Network Working Group

Request for Comments: 4119

J. Peterson

Neustar

Category: Standards Track

December 2005

\title{
A Presence-based GEOPRIV Location Object Format
}

Status of This Memo

This document specifies an Internet standards track protocol for the Internet community, and requests discussion and suggestions for improvements. Please refer to the current edition of the "Internet Official Protocol standards" (STD 1) for the standardization state and status of this protocol. Distribution of this memo is unlimited.

Copyright Notice

Copyright (C) The Internet Society (2005).

Abstract

This document describes an object format for carrying geographical information on the Internet. This location object extends the Presence Information Data Format (PIDF), which was designed for communicating privacy-sensitive presence information and which has similar properties. 
Table of Contents

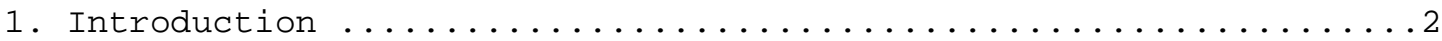

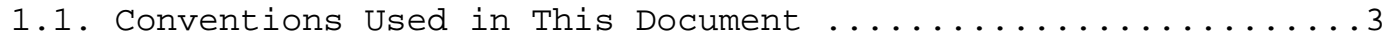

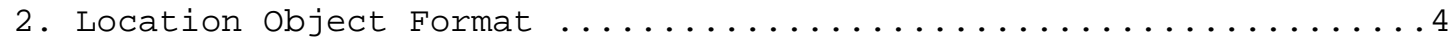



2.2. Extensions to PIDF for Location and Usage Rules .........5

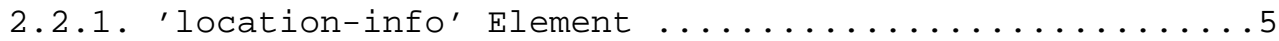



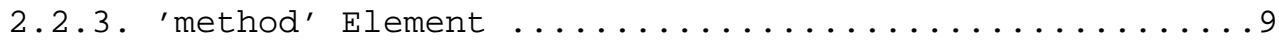

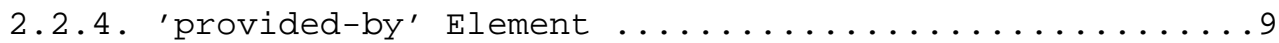

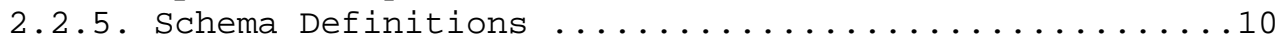

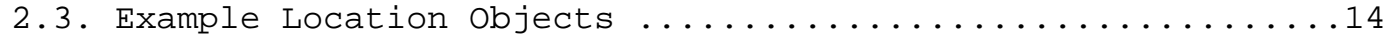

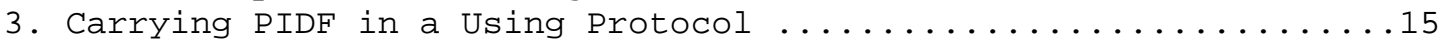

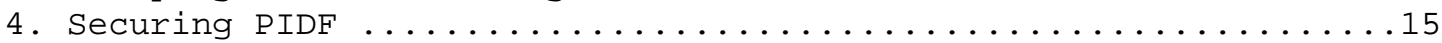

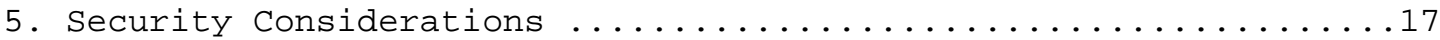



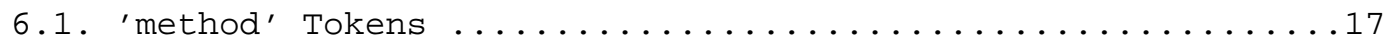

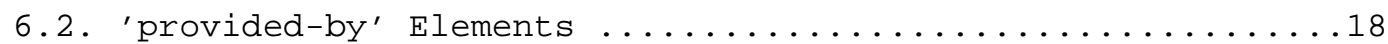

6.3. URN Sub-Namespace Registration for





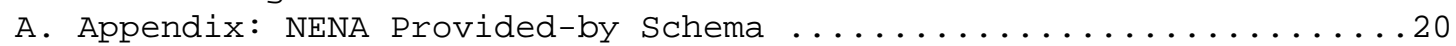



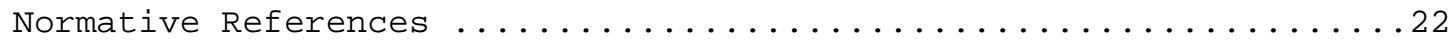

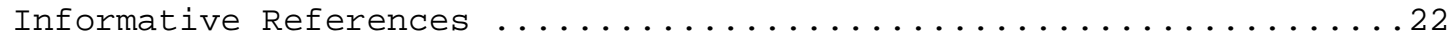

1. Introduction

Geographical location information describes a physical position in the world that may correspond to the past, present, or future location of a person, event, or device. Numerous applications used in the Internet today benefit from sharing location information (including mapping/navigation applications, 'friend finders' on cell phones, and so on). However, such applications may disclose the whereabouts of a person in a manner contrary to the user's preferences. Privacy lapses may result from poor protocol security (which permits eavesdroppers to capture location information), inability to articulate or accommodate user preferences, or similar defects common in existing systems. The privacy concerns surrounding the unwanted disclosure of a person's physical location are among the more serious issues that confront users on the Internet.

Consequently, a need has been identified to convey geographical location information within an object that includes a user's privacy and disclosure preferences and which is protected by strong cryptographic security. Previous work [13] has observed that this problem bears some resemblance to the general problem of 
communicating and securing presence information on the Internet. Presence (defined in [12]) provides a real-time communications disposition for a user, and thus has similar requirements for selective distribution and security.

Therefore, this document extends the XML-based Presence Information Data Format (PIDF [2]) to allow the encapsulation of location information within a presence document.

This document does not invent any format for location information itself. Numerous existing formats based on civic location, geographic coordinates, and the like, have been developed in other standards fora. Instead, this document defines an object that is suitable both for identifying and encapsulating preexisting location information formats, and for providing adequate security and policy controls to regulate the distribution of location information over the Internet.

The location object described in this document can be used independently of any 'using protocol', as the term is defined in the GEOPRIV requirements [10]. It is considered an advantage of this proposal that existing presence protocols (such as [14]) would natively accommodate the location object format defined in this document, and be capable of composing location information with other presence information, because this location object is an extension of PIDF. However, the usage of this location object format is not limited to presence-using protocols-- any protocol that can carry XML or MIME types can carry PIDF.

Some of the requirements in [10] and [11] concern data collection and usage policies associated with location objects. This document provides only the minimum markup necessary for a user to express the necessary privacy preferences as specified by the GEOPRIV requirements (the three basic elements in [11]). However, this document does not demonstrate how a full XML-based ruleset, accommodating the needs of Location Servers, could be embedded in PIDF. It is assumed that other protocols (such as HTTP) will be used to move rules between Rule Holders and Location Servers, and that full rulesets will be defined in a separate document.

1.1. Conventions Used in This Document

The key words "MUST", "MUST NOT", "REQUIRED", "SHALL", "SHALL NOT", "SHOULD", "SHOULD NOT", "RECOMMENDED", "MAY", and "OPTIONAL" in this document are to be interpreted as described in [1]. 


\section{Location Object Format}

\subsection{Baseline PIDF Usage}

The GEOPRIV requirements [10] (or REQ for short) specify the need for a name for the person, place or thing that location information describes (REQ 2.1). PIDF has such an identifier already: every PIDF document has an "entity" attribute of the 'presence' element that signifies the URI of the entity whose presence the document describes. Consequently, if location information is contained in a PIDF document, the URI in the "entity" attribute of the 'presence' element indicates the target of that location information (the 'presentity'). The URI in the "entity" attribute generally uses the "pres" URI scheme defined in [3]. Such URIs can serve as unlinkable pseudonyms (per REQ 12).

PIDF optionally contains a 'contact' element that provides a URI where the presentity can be reached by some means of communication. Usually, the URI scheme in the value of the 'contact' element gives some sense of how the presentity can be reached; if it uses the SIP URI scheme, for example, SIP can be used, and so on. Location information can be provided without any associated means of communication. Thus, the 'contact' element may or may not be present, as desired by the creator of the PIDF document.

PIDF optionally contains a 'timestamp' element that designates the time at which the PIDF document was created. This element corresponds to REQ 2.7a.

PIDF contains a 'status' element, which is mandatory. 'status' contains an optional child element, 'basic', that describes the presentity's communications disposition (in very broad terms: either OPEN or CLOSED). For the purposes of this document, it is not necessary for 'basic' status to be included. If, however, communications disposition is included in a PIDF document above and beyond geolocation, then 'basic' status may appear in a PIDF document that uses these extensions.

PIDF also contains a 'tuple' umbrella element, which holds an "id" element used to uniquely identify a segment of presence information so that changes to this information can be tracked over time (as multiple notifications of presence are received). 'timestamp', 'status', and 'contact' are composed under 'tuple'. 


\subsection{Extensions to PIDF for Location and Usage Rules}

This XML Schema extends the 'status' element of PIDF with a complex element called 'geopriv'. There are two major subelements that are encapsulated within geopriv: one for location information, and one for usage rules. Both of these subelements are mandatory, and are described in subsequent sections. By composing these two subelements under 'geopriv', the usage rules are clearly and explicitly associated with the location information.

For extensibility (see REQ 1.4), the schema allows any other subelements to appear under the 'geopriv' element. Two other optional subelements are included in this document: one that indicates the method by which geographical location was determined, and one that allows an explicit designation of the entity that provided the information.

\subsection{1. 'location-info' Element}

Each 'geopriv' element MUST contain one 'location-info' element. A 'location-info' element consists of one or more chunks of location information (per REQ 2.5). The format of the location information (REQ 2.6) is identified by the imported XML Schema, which describes the namespace in question. All PIDF documents that contain a 'geopriv' element MUST contain one or more import directives indicating the XML Schema(s) that are used for geographic location formats.

In order to ensure interoperability of GEOPRIV implementations, it is necessary to select a baseline location format that all compliant implementations support (see REQ 3.1). Because it satisfies REQ 2.5.1, this document works from the assumption that Geography Markup Language (GML) 3.0 [15] shall be this mandatory format (a MUST implement for all PIDF implementations supporting the 'geopriv' element).

GML is an extraordinarily thorough and versatile system for modeling all manner of geographic object types, topologies, metadata, coordinate reference systems, and units of measurement. The simplest package for GML supporting location

information is the 'feature.xsd' schema. Although 'feature.xsd' can express complicated geographical concepts, it requires very little markup to provide basic coordinate points for the most commonly used cases. Various format descriptions (including latitude/longitude based location information) are supported by Feature (see section 7.4.1.4 of [15] for examples), which resides here: 
urn:opengis: specification: gml:schema-xsd:feature:v3.0

Note that by importing the Feature schema, necessary GML baseline schemas are transitively imported.

Complex features (such as modeling topologies and polygons, directions and vectors, temporal indications of the time for which a particular location is valid for a target) are also available in GML, but require importing additional schemas. For the purposes of baseline interoperability as defined by this document, only support for the 'feature.xsd' GML schema is REQUIRED.

Implementations MAY support the civic location format (civicLoc) defined in section 2.2.5. CivicLoc provides the following elements:

\begin{tabular}{|c|c|c|}
\hline Label & Description & Example \\
\hline country & $\begin{array}{l}\text { The country is } \\
\text { identified by the } \\
\text { two-letter ISO } 3166 \\
\text { code. }\end{array}$ & US \\
\hline $\mathrm{A} 1$ & $\begin{array}{l}\text { national } \\
\text { subdivisions (state, } \\
\text { region, province, } \\
\text { prefecture) }\end{array}$ & New York \\
\hline A2 & $\begin{array}{l}\text { county, parish, gun } \\
\text { (JP), district (IN) }\end{array}$ & King's County \\
\hline A3 & $\begin{array}{l}\text { city, township, shi } \\
\text { (JP) }\end{array}$ & New York \\
\hline A4 & $\begin{array}{l}\text { city division, } \\
\text { borough, city } \\
\text { district, ward, chou } \\
\text { (JP) }\end{array}$ & Manhattan \\
\hline A5 & neighborhood, block & Morningside Heights \\
\hline A 6 & street & Broadway \\
\hline $\mathrm{PRD}$ & $\begin{array}{l}\text { Leading street } \\
\text { direction }\end{array}$ & $\mathrm{N}, \quad \mathrm{W}$ \\
\hline POD & $\begin{array}{l}\text { Trailing street } \\
\text { suffix }\end{array}$ & SW \\
\hline
\end{tabular}




\begin{tabular}{|c|c|c|}
\hline STS & Street suffix & $\begin{array}{l}\text { Avenue, Platz, } \\
\text { Street }\end{array}$ \\
\hline HNO & $\begin{array}{l}\text { House number, } \\
\text { numeric part only. }\end{array}$ & 123 \\
\hline HNS & House number suffix & A, $1 / 2$ \\
\hline LMK & $\begin{array}{l}\text { Landmark or vanity } \\
\text { address }\end{array}$ & Low Library \\
\hline LOC & $\begin{array}{l}\text { Additional location } \\
\text { information }\end{array}$ & Room 543 \\
\hline FLR & Floor & 5 \\
\hline NAM & $\begin{array}{l}\text { Name (residence, } \\
\text { business or office } \\
\text { occupant) }\end{array}$ & Joe's Barbershop \\
\hline $\mathrm{PC}$ & Postal code & $10027-0401$ \\
\hline
\end{tabular}

Either the GML 3.0 geographical information format element, or the location format element ('civicLoc') defined in this document, MAY appear in a 'location-info' element. Both MAY also be used in the same 'location-info' element. In summary, the feature.xsd schema of GML 3.0 MUST be supported by implementations compliant with this specification, and the civicLoc format MAY be supported by implementations compliant with this specification.

\subsection{2. 'usage-rules' Element}

At the time this document was written, the policy requirements for GEOPRIV objects were not definitively completed. However, the 'usage-rules' element exists to satisfy REQ 2.8 and the requirements of the GEOPRIV policy requirements [11] document. Each 'geopriv' element MUST contain one 'usage-rules' element, even if the Rule

Maker has requested that all subelements be given their default values.

Following the policy requirements document (Section 3.1), there are three fields that need to be expressible in Location objects throughout their lifecycle (from Generator to Recipient): one field that limits retransmission, one that limits retention, and one that contains a reference to external rulesets. Those three fields are 
instantiated here by the first three elements. The fourth element provides a generic space for human-readable policy directives. Any of these fields MAY be present in a Location Object 'usage-rules' element; none are required to be.

'retransmission-allowed': When the value of this element is 'no', the Recipient of this Location object is not permitted to share the enclosed Location Information, or the object as a whole, with other parties. When the value of this element is 'yes', distributing this Location is permitted (barring an existing outof-band agreement or obligation to the contrary). By default, the value MUST be assumed to be 'no'. Implementations MUST include this field, with a value of 'no', if the Rule Maker specifies no preference.

'retention-expires': This field specifies an absolute date at which time the Recipient is no longer permitted to possess the location information and its encapsulating Location object; both may be retained only until the time specified by this field. By default, the value MUST be assumed to be twenty-four hours from the 'timestamp' element in the PIDF document, if present; if the 'timestamp' element is also not present, then the value MUST be assumed to be twenty-four hours from the time at which the Location object is received by the Location Recipient. If the value in the 'retention-expires' element has already passed when the Location Recipient receives the Location object, the Recipient MUST discard the Location object immediately.

'ruleset-reference': This field contains a URI that indicates where a fuller ruleset of policies, related to this object, can be found. This URI SHOULD use the HTTPS URI scheme; and if it does, the server that holds these rules MUST authenticate any attempt to access these rules. Usage rules themselves may divulge private information about a Target or Rule Maker. The URI MAY, alternatively, use the CID URI scheme [7], in which case it MUST denote a MIME body carried with the Location object by the using protocol. Rulesets carried as MIME bodies SHOULD be encrypted and signed by the Rule Maker; unsigned rulesets SHOULD NOT be honored by Location Servers or Location Recipients. Note that in order to avoid network lookups that result in an authorization failure, creators of Location Objects MAY put HTTPS-based rulesetreferences into an encrypted external MIME body referenced by a CID; in this way, recipients of the Location object that are unable to decrypt the external MIME body will not learn the HTTPS URI unless they are able to decrypt the MIME body. 
'note-well': This field contains a block of text containing further generic privacy directives. These directives are intended to be human-readable only, not to be processed by any automaton.

\subsection{3. 'method' Element}

The optional 'method' element describes the way that the location information was derived or discovered. An example of this element (for a geographical position system) is:

$<$ method $>$ gps $</$ method $>$

The possible values of the 'method' element are enumerated within an IANA registry. Implementations MUST limit the use of this method to the values shepherded by IANA. This document pre-populates the IANA registry with seven possible values; see section 6.1 for more information.

The 'method' element is useful, for example, when multiple sources are reporting location information for a given user, and some means of determining location might be considered more authoritative than others (i.e., a dynamic, real-time position system versus static provisioning associated with a target device). However, note that inclusion of 'method' might reveal sensitive information when the generator is providing intentionally coarsened location information. For example, when a LO is transmitted with 'DHCP' as the 'method', but the location information indicates only the city in which the generator is located, the sender has good justification to suspect that some location information is being withheld.

\subsection{4. 'provided-by' Element}

The optional 'provided-by' element describes the entity or organization that supplied this location information (beyond the domain information that can be inferred from a signing certificate). An example of this element (for a made-up game system) might be:

$<$ provided-by $>$

$<$ test:game> West 5

$</$ test: game $>$

$</$ provided-by $>$

Values for the 'provided-by' element MUST be IANA-registered XML namespaces; see Section 6.2 for more information. 
The 'provided-by' element is not intended for use by most entities, but rather to meet special requirements for which overhead (IANA registration, location object size) and potential location information leakage are acceptable choices.

In general cases, the entity that supplied location information is communicated by the subjectAltName of the certificate with which the location object is signed; thus, this element is unnecessary.

'Provided-by' is meaningful in particular cases when the creator of a location object wants to designate a particular system or party within a complex administrative domain, including situations envisioned for providing emergency services in a diverse national context. It might assist, for example, the recipient of a malformed or misleading location object in identifying the particular system that malfunctioned.

Users should be aware that this information can inadvertently provide additional information to the receiver, increasing the effective resolution of the geospatial or civic information, or even revealing some location information, when it was meant to be entirely protected. Consider if there were circumstances that influenced Columbia University to elect to register and use the provided-by element. If an example Lo includes only state-level information, then including the fact that the location information was provided by Columbia University provides a strong indication that the Target is actually located in a four-block area in Manhattan. Accordingly, this element should be used only when organizational functions strongly would depend on it. In all but such usages, the subjectAltName of the certificate will suffice, and 'provided-by' SHOULD NOT be used.

\subsubsection{Schema Definitions}

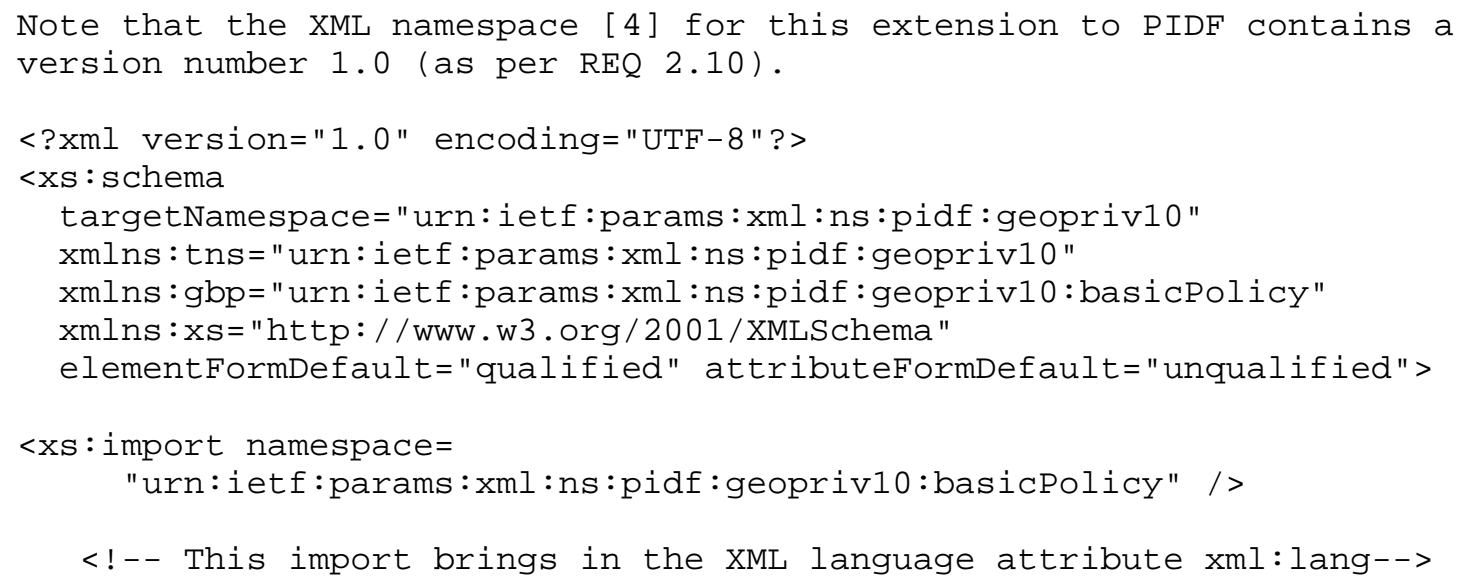




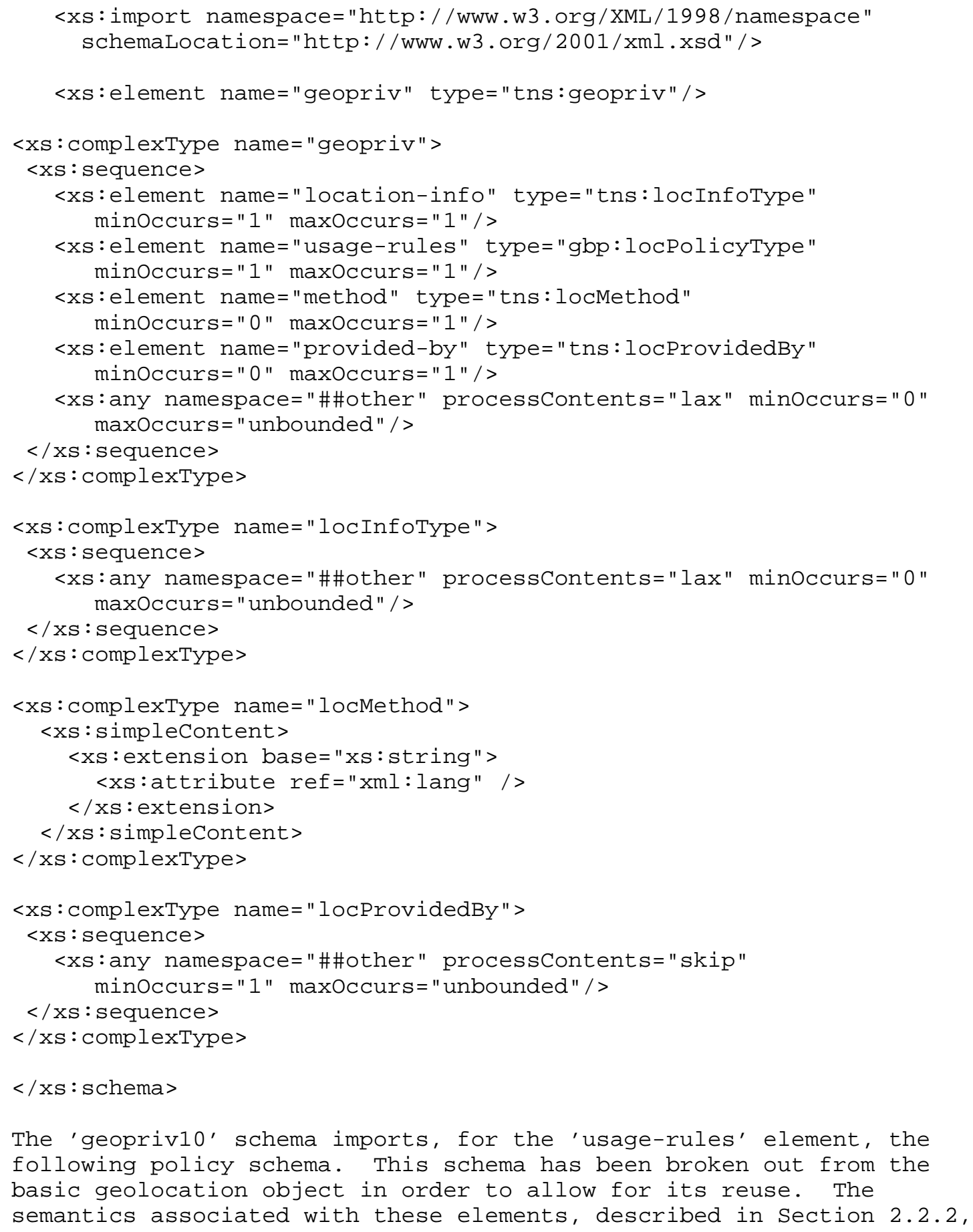




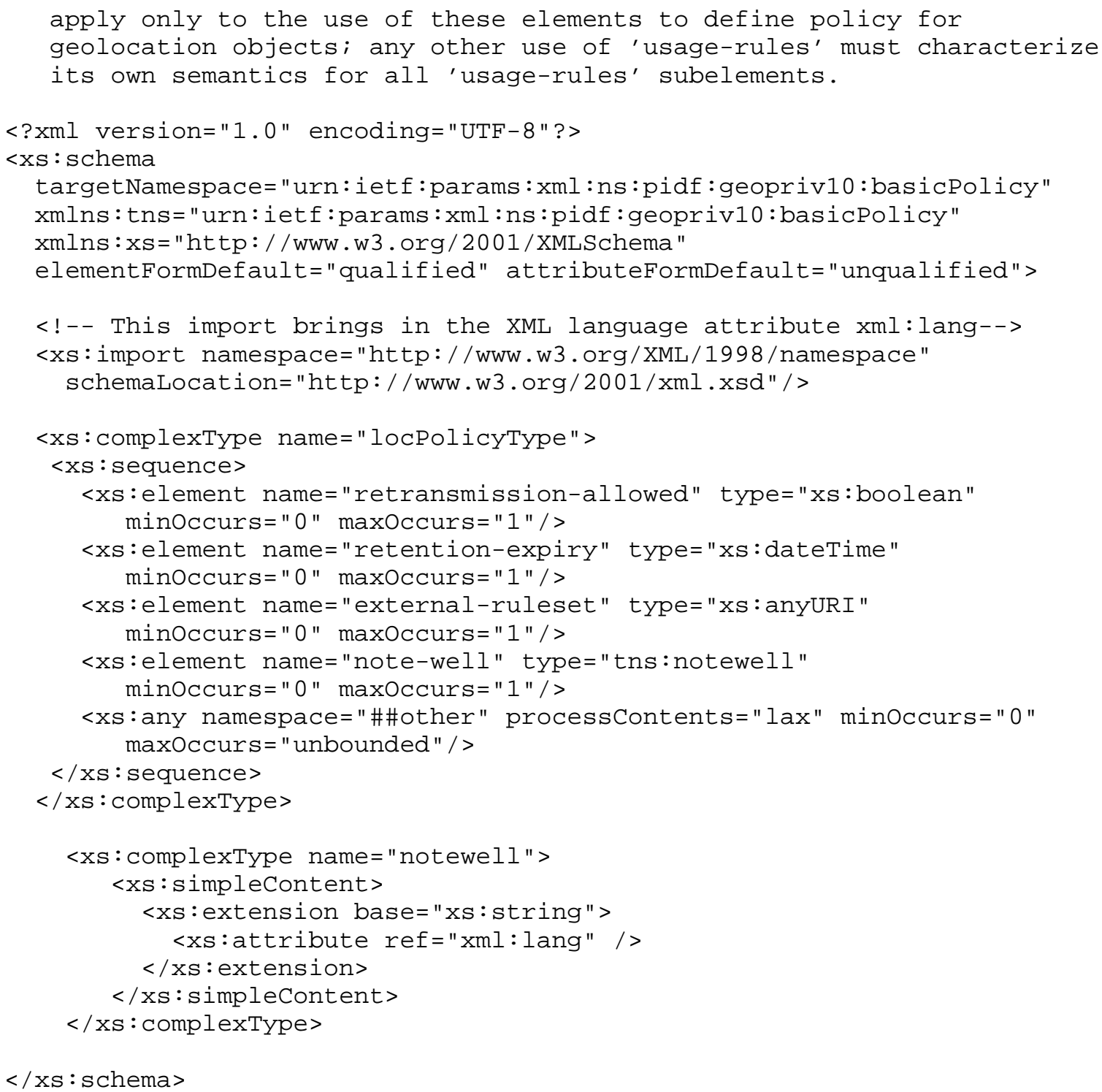

The following schema is a trivial representation of civic location that MAY be implemented by entities compliant with this specification.

$<$ ?xml version="1.0" encoding="UTF-8"?> 


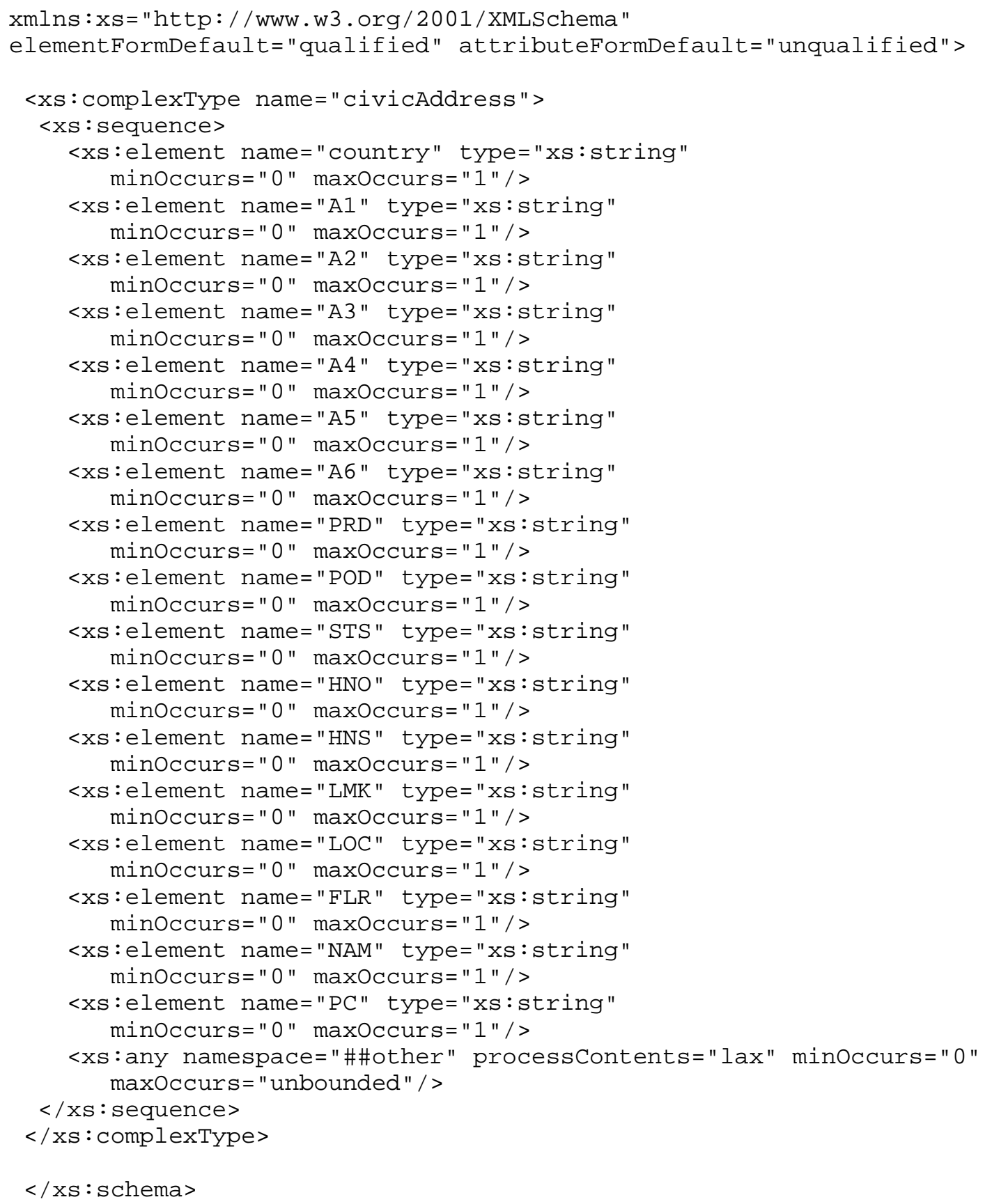




\subsection{Example Location Objects}

Note that these examples show PIDF documents without any MIME headers or security applied to them (see section 4 below).

The following XML instance document is an example of the use of a simple GML 3.0 markup with a few of the policy directives specified above within a PIDF document. The GPS coordinates given in the 'gml' element are for San Francisco, CA.

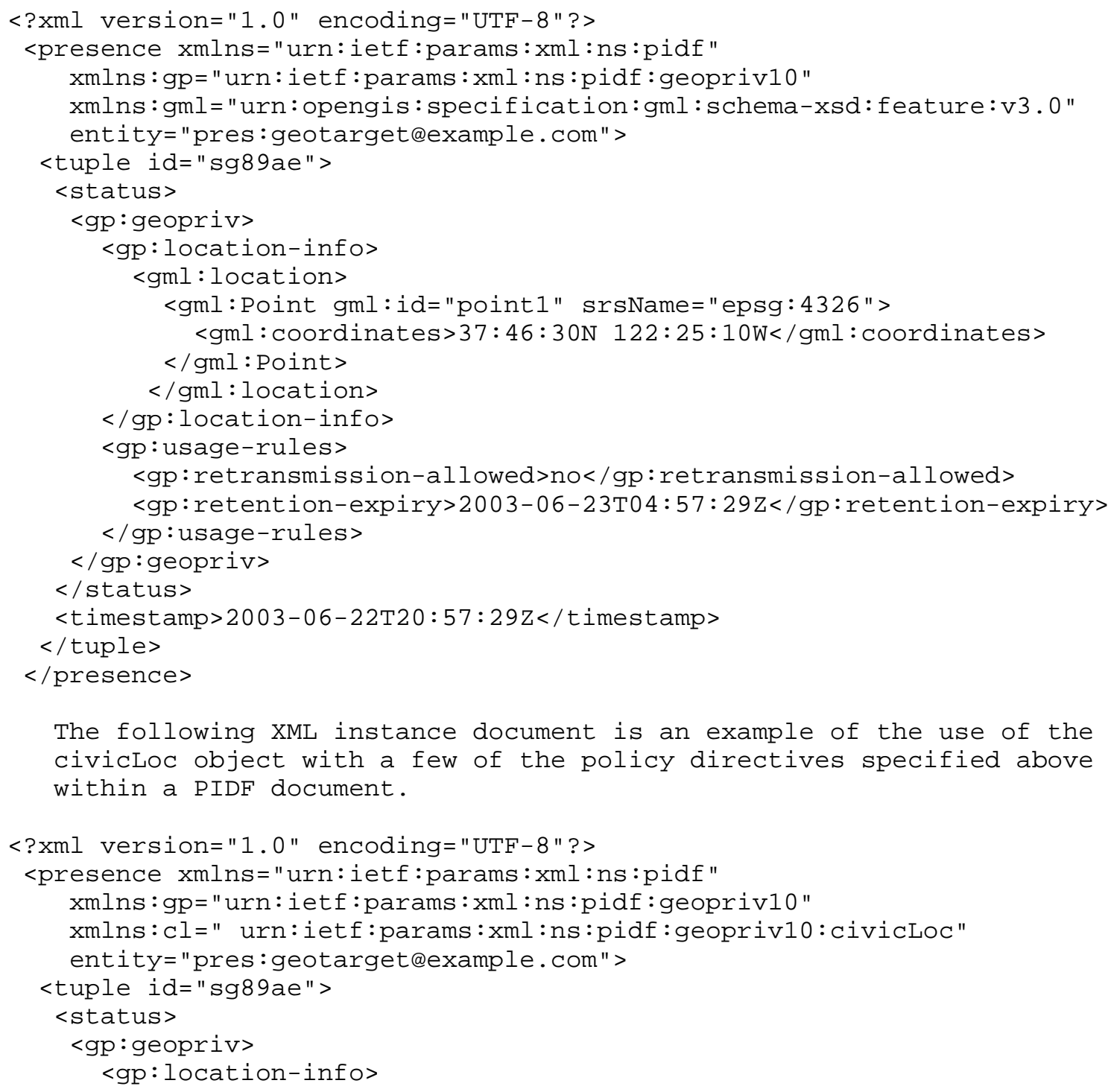

The following XML instance document is an example of the use of the civicLoc object with a few of the policy directives specified above within a PIDF document. 


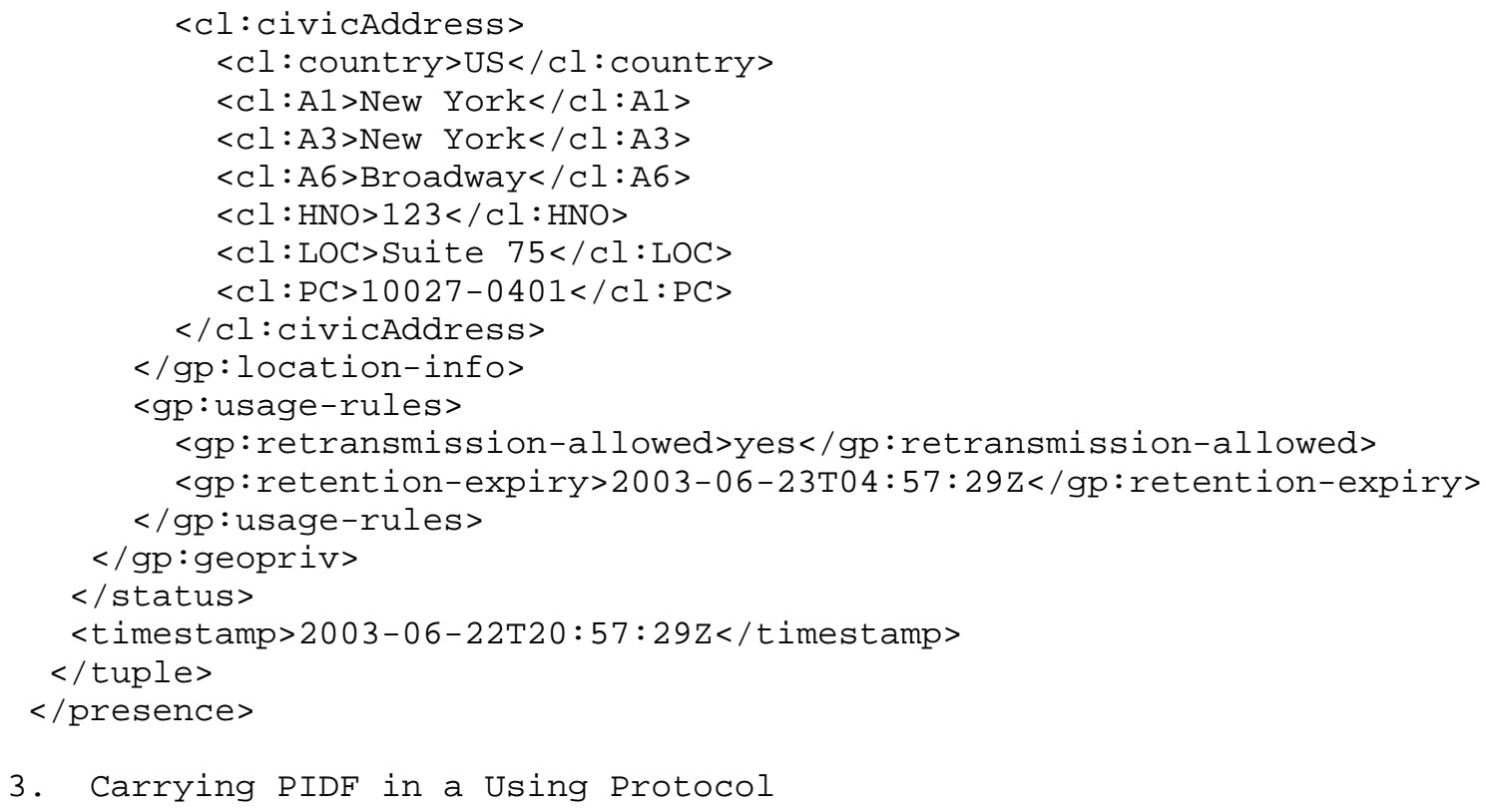

A PIDF document is an XML document; therefore, PIDF might be carried in any protocol capable of carrying XML. A MIME type has also been registered for PIDF: 'application/pidf+xml'. PIDF may therefore be carried as a MIME body in protocols that use MIME (such as SMTP, HTTP, or SIP) with an encapsulating set of MIME headers, including a Content-Type of 'application/pidf+xml'.

Further specification of the behavior of using protocols (including subscribing to or requesting presence information) is outside the scope of this document.

\section{Securing PIDF}

There are a number of ways in which XML documents can be secured. XML itself supports several ways of partially securing documents, including element-level encryption and digital signature properties.

For the purposes of this document, only the securing of a PIDF document as a whole, rather than element-by-element security, is considered. None of the requirements [10] suggest that only part of the information in a location object might need to be protected while other parts are unprotected; virtually any such configuration would introduce potentials for privacy leakage. Consequently, the use of MIME-level security is appropriate. 
S/MIME [5] allows security properties (including confidentiality, integrity, and authentication properties) to be applied to the contents of a MIME body. Therefore, all PIDF implementations that support the XML Schema extensions for location information described in this document MUST support S/MIME; in particular, they MUST support the CMS [6] EnvelopedData and SignedData content types, which are used for encryption and digital signatures, respectively. It is believed that this mechanism meets REQs 2.10, 13, 14.1, 14.2, 14.3, and 14.4 .

Additionally, all compliant applications MUST implement the AES encryption algorithm for S/MIME, as specified in [8] (and per REQ 15.1). Of course, implementations MUST also support the baseline encryption and digital signature algorithms described in the S/MIME specification.

S/MIME generally entails the use of X.509 [9] certificates. In order to encrypt a request for a particular destination end-to-end (i.e., to a Location Recipient), the Location Generator must possess credentials (typically an X.509 certificate) that have been issued to the Location Recipient. Implementations of this specification SHOULD support X.509 certificates for S/MIME, and MUST support passwordbased CMS encryption (see [6]). Any symmetric keying systems SHOULD derive high-entropy content encoding keys (CEKs). When X.509 certificates are used to sign PIDF Location Objects, the subjectAltName of the certificate SHOULD use the "pres" URI scheme.

One envisioned deployment model for S/MIME in PIDF documents is the following. Location Servers hold X.509 certificates and share secrets with Location Generators and Location Recipients. When a Generator sends location information to a Server, it can be encrypted with S/MIME (or any lower-layer encryption specific to the using protocol). When a server forwards location information to a Recipient, location information can be encrypted with password-based CMS encryption. This allows the use of encryption when the Location Recipient does not possess its own X.509 certificate.

S/MIME was designed for end-to-end security between email peers that communicate through multiple servers (i.e mail transfer agents) that do not modify message bodies. There is, however, at least one instance in which Location Servers modify Location objects: when Location Servers enforce policies on behalf of the Rule Maker. For example, a Rule Maker may specify that Location Information should be coarsened (made less specific) before it is transmitted to particular recipients. If the Location Server were unable to modify a Location object, because it was encrypted, signed, or both, it would be unable to accomplish this function. Consequently, when a Location Generator wants to allow a Location server to modify such messages, they MAY 
encrypt such messages with a key that can be decrypted by the Location Server (the digital signature, of course, can still be created with keying material from the Location Generator's certificate). After modifying the Location Object, the Location

Server can re-sign the object with its own credentials (encrypting it with any keys issued to the Location Recipient, if they are known to the server).

Note that policies for data collection and usage of location information, in so far as they are carried within a location object, are discussed in section 2.2.2.

5. Security Considerations

The threats facing an Internet protocol that carries geolocation information are detailed in [16]. The requirements that were identified in that analysis of the threat model were incorporated into [10], in particular within section 7.4. This document aims to be compliant with the security requirements derived from those two undertakings, in so far as they apply to the location object itself (as opposed to the using protocol).

Security of the location object defined in this document, including normative requirements for implementations, is discussed in section 4. This security focuses on end-to-end integrity and confidentiality properties that are applied to a location object for its lifetime via S/MIME.

Security requirements associated with using protocols (including authentication of subscribers to geographical information, etc.) are outside the scope of this document.

6. IANA Considerations

6.1. 'method' Tokens

This document requests that the IANA create a new registry for 'method' tokens associated with the PIDF-LO object. 'method' tokens are text strings designating the manner in which location information in a PIDF-LO object has been derived or discovered. Any party may register new 'method' tokens with the IANA, as needed, on a firstcome-first-serve basis.

This section pre-registers 7 new 'method' tokens associated with the 'method' element described above in section 2.2.3: 
GPS: Global Positioning System

A-GPS: GPS with assistance

Manual: entered manually by an operator or user, e.g., based on subscriber billing or service location information

DHCP: provided by DHCP (used for wireline access networks, see 802.11 below)

Triangulation: triangulated from time-of-arrival, signal strength, or similar measurements

Cell: location of the cellular radio antenna

802.11: 802.11 access point (used for DHCP-based provisioning over wireless access networks)

\section{2. 'provided-by' Elements}

This document requests that IANA create a new registry of XML namespaces for 'provided-by' elements for use with PIDF-LO objects. Registrations of new XML namespaces that are used for 'provided-by' MUST be reviewed by an Expert Reviewer designated by the IESG.

This document pre-registers a single XML namespace for 'provided-by', which is given in Appendix A.

6.3. URN Sub-Namespace Registration for

urn:ietf:params: $x m l: n s: p i d f:$ geopriv10

This section registers a new XML namespace, as per the guidelines in [4].

URI: The URI for this namespace is urn: ietf:params:xml:ns:pidf:geopriv10.

Registrant Contact: IETF, GEOPRIV working group, (geoprivaietf.org), Jon Peterson (jon.peterson@neustar.biz). XML :

\section{BEGIN}

$<$ ?xml version="1.0"?>

<!DOCTYPE html PUBLIC "-//W3C//DTD XHTML Basic 1.0//EN"

"http://www.w3.org/TR/xhtml-basic/xhtml-basic10.dtd">

<html xmlns="http://www.w3.org/1999/xhtml">

$<$ head $>$

$<$ meta http-equiv="content-type"

content="text/html; charset=iso-8859-1" />

<title>GEOPRIV PIDF Extensions</title>

$</$ head $>$

$<$ body $>$

$<$ h1>PIDF Extensions of Geographical Information and Privacy</h1>

$<$ h2>urn: ietf:params:xml:ns:pidf:geopriv10</h2>

<p>See <a href="ftp://ftp.rfc-editor.org/in-notes/rfc4119.txt"> 
$</$ body $>$

$\operatorname{RFC} 4119</ \mathrm{a}\rangle .</ \mathrm{p}>$

$</$ html $>$

END

7. Acknowledgements

This document was produced with the assistance of many members of the GEOPRIV IETF working group. Special thanks to Carl Reed of OpengIS for a close read of the document.

The civic location format described in this document was proposed by Henning Schulzrinne for communicating location information in DHCP, and has been appropriated in its entirety for this document.

James M. Polk provided the text related to the 'method' element, and much of the text for the 'provided-by' element. The text of Appendix A was written by Nadine Abbott. 
A. Appendix: NENA Provided-By Schema

The following registers the XML namespace urn:ietf:params:xml:ns:pidf:geopriv10:dataProvider and the associated schema below, for usage within the 'provided-by' element of PIDF-LO. The dataProvider namespace was developed by the US National Emergency Number Administration (NENA) for next-generation emergency communications needs.

This appendix is non-normative for implementers of PIDF-LO implementations and MAY support the dataProvider namespace. Other registrants of 'provided-by' namespaces are invited to use the registration below as an informative example.

URI: The URI for this namespace is

urn: ietf:params: $x m 1: n s: p i d f:$ geopriv10:dataProvider

Registrant Contact: NENA, VoIP working group \& IETF, GEOPRIV

working group, (geopriv@ietf.org), Nadine Abbott

(nabbottetelcordia.com).

XML :

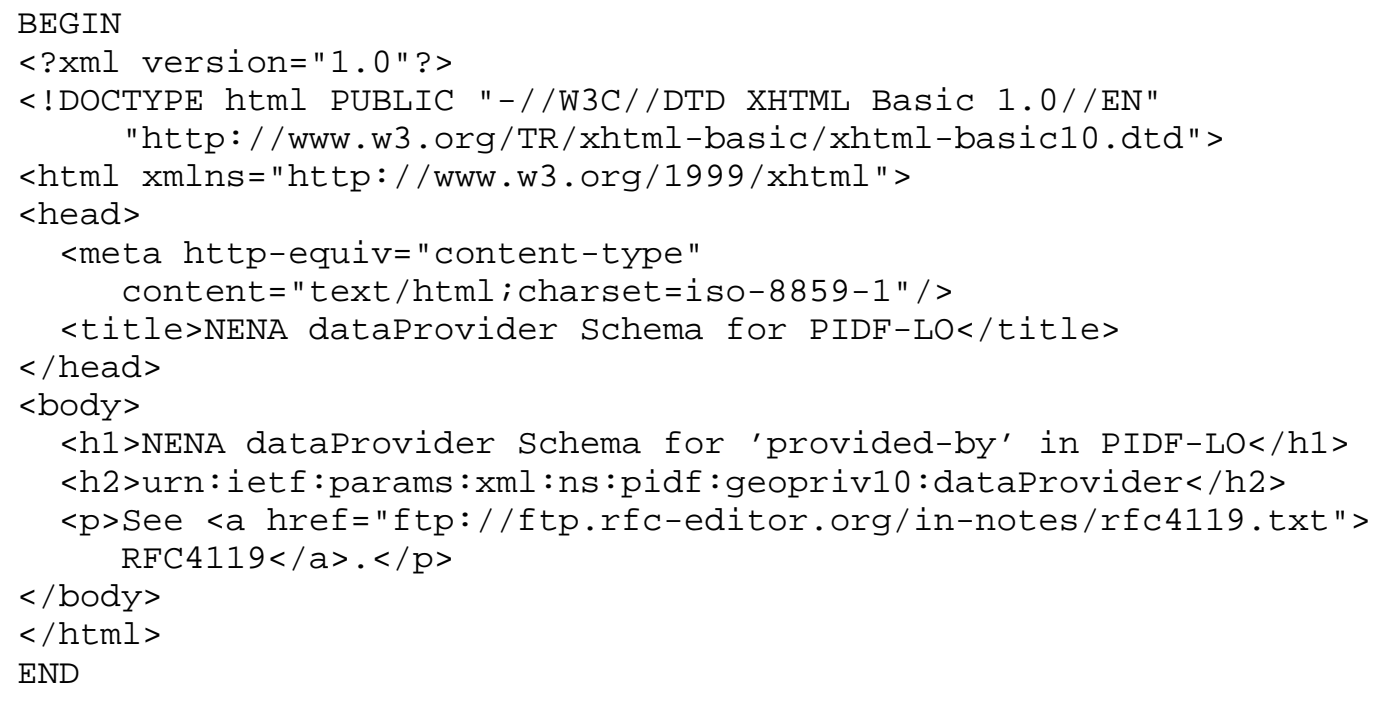




\section{A.1. dataProvider XML Schema}

$<$ ?xml version="1.0" encoding="UTF-8"?>

<!-- edited with XMLSPY v5 rel. 3 U (http://www.xmlspy.com) by

Patricia Bluhm (HBF Group) -->

<xs: schema

targetNamespace="urn: ietf :params: xml:ns:pidf : geopriv10:dataProvider"

xmlns:tns="urn: ietf:params: xml:ns:pidf:geopriv10:dataProvider"

xmlns:xs="http://www.w3.org/2001/XMLSchema"

elementFormDefault="qualified" attributeFormDefault="unqualified" >

<xs:element name="nena" type="tns:DataProviderIDType"/>

<xs:complextype name="DataProviderIDType">

$<x$ : annotation>

$<x$ : documentation>NENA registered Company ID

for Service Provider supplying location information</xs:documentation $>$

$</ x s$ : annotation $>$

$<x s: a l l>$

$<x$ :element name="DataProviderID"

type="tns: NENACompanyIDType" minOccurs $=" 0 " />$

$<x s: e l e m e n t$ name $="$ TelURI"

type="tns:TelURI_24x7Type" minOccurs="0"/>

minoccurs $=" 0 " />$

$<x s: e l e m e n t$ name="URL" type="xs:anyURI"

$</ x$ :all $>$

$</ x s$ : complextype $>$

<xs:simpleType name="NENACompanyIDType">

<xs:annotation>

<xs: documentation>NENA registered Company

ID. $</ x s:$ documentation $>$

$</ x$ : annotation $>$

<xs:restriction base="xs:string">

$<\mathrm{xs}$ :maxLength value=" $5 " />$

$</ x$ : restriction $>$

$</ x$ : simpletype $>$

<xs:simpleType name="TelURI_24x7Type">

$<x s$ :annotation>

<xs: documentation>24x7 Tel URI for the

caller's [location data] service provider. To be used for contacting service provider to resolve problems with location data. Possible values TN number, enumerated values when not

available.</xs: documentation>

$</ x$ : annotation>

<xs:union memberTypes="xs:anyURI">

$<x s$ : simpleType>

<xs:restriction base="xs:string">

$<x s:$ maxlength value="10"/>

$<x$ : enumeration value="NOT FOUND"/>

Peterson

Standards Track

[Page 21] 
$</ x s$ :union $>$

$</ x s$ : simpletype $>$

$</ x s$ : schema $>$

<xs:enumeration value="UNAVAILABLE" / > $</ x s$ :restriction $>$

$</ x s:$ simpletype $>$

Normative References

[1] Bradner, S., "Key words for use in RFCs to indicate requirement levels", BCP 14, RFC 2119, March 1997.

[2] Sugano, H., Fujimoto, S., Klyne, G., Bateman, A., Carr, W., and J. Peterson, "Presence Information Data Format (PIDF)", RFC 3863, August 2004 .

[3] Peterson, J., "Common Profile for Presence (CPP)", RFC 3859, October 2003.

[4] Mealling, M., "The IETF XML Registry", RFC 3688, BCP 81, January 2004 .

[5] Ramsdell, B., "Secure/Multipurpose Internet Mail Extensions (S/MIME) Version 3.1 Message Specification", RFC 3851, July 2004 .

[6] Housley, R., "Cryptographic Message Syntax (CMS)", RFC 3852, July 2004 .

[7] Levinson, E., "Content-ID and Message-ID Uniform Resource Locators", RFC 2392, August 1998.

[8] Schaad, J., "Use of the Advanced Encryption Standard (AES) Encryption Algorithm in Cryptographic Message Syntax (CMS) ", RFC 3565, July 2003 .

[9] Ramsdell, B., "Secure/Multipurpose Internet Mail Extensions (S/MIME) Version 3.1 Certificate Handling", RFC 3850, July 2004.

Informative References

[10] Cuellar, J., Morris, J., Mulligan, D., Peterson, J., and J. Polk, "Geopriv Requirements", RFC 3693, February 2004.

[11] Morris, J., Mulligan, D., and J. Cuellar, "Core Privacy Protections for Geopriv Location Object", Work in Progress, June 2003. 
[12] Day, M., Rosenberg, J., and H. Sugano, "A Model for Presence and Instant Messaging", RFC 2778, February 2000.

[13] Peterson, J., "A Presence Architecture for the Distribution of Geopriv Location Objects", Work in Progress, February 20003.

[14] Rosenberg, J., Schulzrinne, H., Camarillo, G., Johnston, A., Peterson, J., Sparks, R., Handley, M., and E. Schooler, "SIP: Session Initiation Protocol", RFC 3261, May 2002.

[15] OpenGIS, "Open Geography Markup Language (GML) Implementation Specification", OGC 02-023r4, January 2003, <http://www.opengeospatial.org/specs/?page=specs>.

[16] Danley, M., Mulligan, D., Morris, J., and J. Peterson, "Threat Analysis of the Geopriv Protocol", RFC 3694, February 2004.

Author's Address

Jon Peterson

Neustar, Inc.

1800 sutter st

Suite 570

Concord, CA 94520

US

Phone: +1 925/363-8720

EMail: jon.petersoneneustar.biz

URI: http://www.neustar.biz/ 
Full Copyright statement

Copyright (C) The Internet Society (2005).

This document is subject to the rights, licenses and restrictions contained in BCP 78, and except as set forth therein, the authors retain all their rights.

This document and the information contained herein are provided on an "AS IS" basis and THE CONTRIBUTOR, THE ORGANIZATION HE/SHE REPRESENTS OR IS SPONSORED BY (IF ANY), THE INTERNET SOCIETY AND THE INTERNET ENGINEERING TASK FORCE DISCLAIM ALL WARRANTIES, EXPRESS OR IMPLIED, INCLUDING BUT NOT LIMITED TO ANY WARRANTY THAT THE USE OF THE INFORMATION HEREIN WILL NOT INFRINGE ANY RIGHTS OR ANY IMPLIED WARRANTIES OF MERCHANTABILITY OR FITNESS FOR A PARTICULAR PURPOSE.

Intellectual Property

The IETF takes no position regarding the validity or scope of any Intellectual Property Rights or other rights that might be claimed to pertain to the implementation or use of the technology described in this document or the extent to which any license under such rights might or might not be available; nor does it represent that it has made any independent effort to identify any such rights. Information on the procedures with respect to rights in RFC documents can be found in BCP 78 and BCP 79 .

Copies of IPR disclosures made to the IETF Secretariat and any assurances of licenses to be made available, or the result of an attempt made to obtain a general license or permission for the use of such proprietary rights by implementers or users of this specification can be obtained from the IETF on-line IPR repository at http://www.ietf.org/ipr.

The IETF invites any interested party to bring to its attention any copyrights, patents or patent applications, or other proprietary rights that may cover technology that may be required to implement this standard. Please address the information to the IETF at ietfipreietf.org.

Acknowledgement

Funding for the RFC Editor function is currently provided by the Internet Society. 